\title{
WELL-POSEDNESS OF THE INITIAL VALUE PROBLEM FOR THE KORTEWEG-DE VRIES EQUATION
}

\author{
CARLOS E. KENIG, GUSTAVO PONCE, AND LUIS VEGA
}

\section{INTRODUCTION}

This paper is mainly concerned with the initial value problem (IVP) for the Korteweg-de Vries (KdV) equation

$$
\left\{\begin{array}{l}
\partial_{t} u+\partial_{x}^{3} u+u \partial_{x} u=0, \quad x, t \in \mathbb{R}, \\
u(x, 0)=u_{0}(x) .
\end{array}\right.
$$

The $\mathrm{KdV}$ equation, which was first derived as a model for unidirectional propagation of nonlinear dispersive long waves [21], has been considered in different contexts, namely in its relation with the inverse scattering method, in plasma physics, and in algebraic geometry (see [24], and references therein).

Our purpose is to study local and global well-posedness of the IVP (1.1) in classical Sobolev spaces $H^{S}(\mathbb{R})$. We shall say that the IVP $(1.1)$ is locally (resp. globally) well-posed in the function space $X$ if it induces a dynamical system on $X$ by generating a continuous local (resp. global) flow.

It was established in the works of Bona and Smith [3], Bona and Scott [2], Saut and Temam [30], and Kato [15] that the IVP (1.1) is locally (resp. globally) well-posed in $H^{s}$ with $s>3 / 2$ (resp. $s \geq 2$ ).

Roughly speaking, global well-posedness in $H^{s}$ depends on the available local theory and on the conservation laws satisfied by solutions of (1.1), namely:

$$
\begin{aligned}
& \Phi_{1}(u)=\int u d x, \quad \Phi_{2}(u)=\int u^{2} d x \\
& \Phi_{3}(u)=\int\left(\left(\partial_{x} u\right)^{2}-\frac{1}{3} u^{3}\right) d x \\
& \Phi_{4}(u)=\int\left(\left(\partial_{x}^{2} u\right)^{2}-\frac{5}{3} u\left(\partial_{x} u\right)^{2}+\frac{5}{36} u^{4}\right) d x \ldots
\end{aligned}
$$

The conservation law $\Phi_{k+2}(\cdot)$ provides an a priori estimate of the $H^{k}-$ norm $(k=0,1, \ldots)$ of a solution with data $u_{0} \in H^{k}$. Hence, combining the local result $(s>3 / 2)$, the invariants $\Phi_{2}-\Phi_{4}$, and some inequalities one obtains that the IVP (1.1) is globally well-posed in $H^{s}$ with $s \geq 2$.

Received by the editors September 20, 1990.

1991 Mathematics Subject Classification. Primary 35Q53; Secondary 35G25, 35D99.

The authors were supported in part by NSF grants. 
On the other hand, as was remarked in [16], the proofs of the local result in $H^{s}$ with $s>3 / 2$ have almost nothing to do with the special structure of the $\mathrm{KdV}$ equation. Indeed, the same proofs work for the inviscid Burgers equation

$$
\partial_{t} u+u \partial_{x} u=0
$$

In [18], we proved that the IVP (1.1) is locally well-posed in $H^{s}$ with $s>9 / 8$. However, none of the global results there apply to the $\mathrm{KdV}$ equation.

The main result in this paper (see Theorems 1.1 and 1.2 below) shows that the IVP (1.1) is locally well-posed in $H^{s}$ with $s>3 / 4$, and consequently globally well-posed in $H^{s}$ with $s \geq 1$. Thus, we answer the questions left open by Saut and Temam [30] (Remark 2.1), and by Kato [16] (§3).

Our method of proof relies heavily on the dispersive character of the $\mathrm{KdV}$ equation. In fact, it can be applied to the IVP

$$
\left\{\begin{array}{l}
\partial_{t} u-D^{\alpha} \partial_{x} u+u^{k} \partial_{x} u=0, \quad x, t \in \mathbb{R}, \\
u(x, 0)=u_{0}(x),
\end{array}\right.
$$

where $D=\left(-\partial_{x}^{2}\right)^{1 / 2}, \alpha \geq 1$, and $k \in Z^{+}$. Thus, for the values $(\alpha, k)=$ $(2,1),(2,2)$, and $(1.1)$ we obtain the $\mathrm{KdV}$ equation, the modified $\mathrm{KdV}$ equation, and the Benjamin-Ono equation $[1,25]$ respectively.

Before stating precisely our result it is convenient to discuss the main ingredients in the proof.

We shall begin by considering the associated linear problem

$$
\left\{\begin{array}{l}
\partial_{t} v-D^{\alpha} \partial_{x} v=0, \quad x, t \in \mathbb{R}, \alpha \geq 1, \\
v(x, 0)=v_{0}(x),
\end{array}\right.
$$

whose solution is given by the unitary group $\left\{U^{\alpha}(t)\right\}_{-\infty}^{\infty}$ in $H^{s}(\mathbb{R})$, where $U^{\alpha}(t)=e^{i t D^{\alpha} \partial_{x}}$ (i.e., $\left.v(x, t)=U^{\alpha}(t) v_{0}(x)\right)$.

Our first tool is the global smoothing effect of Strichartz type [33] present in solutions of (1.3). More precisely, it will be shown (see §2) that

$$
\left(\int_{-\infty}^{\infty}\left\|D^{(\alpha-1) / 4} U^{\alpha}(t) v_{0}\right\|_{\infty}^{4} d t\right)^{1 / 4} \leq c\left\|v_{0}\right\|_{2} .
$$

Next, we have the local smoothing effect first established by Kato [16] (§6) in solutions of the KdV equation. He proved that smooth solutions of the IVP (1.1) satisfy

$$
\int_{-R}^{R} \int_{-T}^{T}\left|\partial_{x} u(x, t)\right|^{2} d t d x \leq c=c\left(R ; T ;\left\|u_{0}\right\|_{2}\right) .
$$

The estimate (1.5) was used in [16] to show existence of a weak solution of the IVP (1.1) with data $u_{0} \in L^{2}(\mathbb{R})$ (see also Kruzhkov and Framinskii [22]).

The corresponding local smoothing effect in the group $\left\{U^{\alpha}(t)\right\}_{-\infty}^{\infty}$ is given by the following identity:

$$
\int_{-\infty}^{\infty}\left|D^{\alpha / 2} U^{\alpha}(t) v_{0}(x)\right|^{2} d t=c_{\alpha}\left\|v_{0}\right\|_{2}^{2}
$$


for any $x \in \mathbb{R}$. Notice that (1.6) is an $L^{\infty}$-estimate in the space variable, which is stronger than the one described in (1.5).

In [8], Constantin and Saut showed that the version of the local smoothing effect described in (1.5) is a common property of linear dispersive equations, where, roughly speaking, the gain of derivatives is equal to $(m-1) / 2$, with $m$ denoting the order. Simultaneously, Sjölin [31] and Vega [36] established a similar result for powers of the Laplacian in their study of the following problem proposed by Carleson [5]: if $u_{0} \in H^{s}\left(\mathbb{R}^{n}\right)$, for which $s$ does

$$
\lim _{t \rightarrow 0} e^{i t \Delta} u_{0}(x)=u_{0}(x) \text { a.e. } x ?
$$

In [19], we proved that the stronger version of this local smoothing effect given in (1.6) (i.e., in the $L^{\infty}\left(\mathbb{R} ; d x: L^{2}(d t)\right)$-norm) holds for any one-dimensional linear dispersive model, and that this result is sharp.

Although, for the sake of completeness, the proofs of (1.4) and (1.6) will be sketched in $\S 2$, we refer to [19] for detailed proofs, extensions of these estimates to general dispersive equations, related results, and further references.

To complement the inequality in (1.6) we shall need some estimates related to the pointwise behavior of $U^{\alpha}(t) v_{0}$. These are again intimately connected to the problem raised by Carleson. From the results of Vega [37] (see also Dahlberg and Kenig [9], Kenig and Ruiz [20], and Sjölin [31]) we have the following bounds for the associated maximal function $\sup _{[-T, T]}\left|U^{\alpha}(t) \cdot\right|$ :

$$
\left(\int_{-\infty[-T, T]}^{\infty} \sup \left|U^{\alpha}(t) v_{0}\right|^{2}(x) d x\right)^{1 / 2} \leq c(1+T)^{\rho}\left\|v_{0}\right\|_{s, 2}
$$

and

$$
\left(\sum_{j=-\infty}^{\infty} \sup _{x \in[j, j+1)} \sup _{[-T, T]}\left|U^{\alpha}(t) v_{0}\right|^{2}(x)\right)^{1 / 2} \leq c(1+T)^{\rho}\left\|v_{0}\right\|_{s, 2},
$$

where $\rho>3 / 4, s>(\alpha+1) / 4$, and $\|\cdot\|_{s, 2}=\left\|(1-\Delta)^{s / 2} \cdot\right\|_{2}$.

In [13], Ginibre and Tsutsumi proved that solutions $u=u(x, t)$ of the IVP (1.1) satisfy

$$
\left(\sum_{j=-\infty}^{\infty} \sup _{[-T, T]} \int_{j}^{j+1} u^{2}(x, t) d x\right)^{1 / 2} \leq C\left(T ;\left\|u_{0}\right\|_{1,2}\right) .
$$

It is interesting to remark that the proof of inequality (1.9) follows the same argument used by Kato in [16] to establish the local smoothing effect (1.5). Notice that the estimate (1.8) is stronger than (1.7) and that this estimate (1.7) when $\alpha=2$ is stronger than (1.9). 
The final ingredient, which suggests the sharpness of our result for the IVP (1.1), is energy estimates. Using the commutator estimates deduced by Kato and Ponce in [17] (see Lemma 2.10) one easily finds that solutions of the IVP (1.1) satisfy

$$
\sup _{[-T, T]}\|u(t)\|_{s, 2} \leq c_{s}\left\|u_{0}\right\|_{s, 2} \exp \left(\int_{-T}^{T}\left\|\partial_{x} u(\tau)\right\|_{\infty} d \tau\right)
$$

for any $s \geq 0$.

From (1.4) it follows that the exponential term in (1.10) when evaluated in $U^{2}(t) v_{0}$ (i.e., the solution of the associated linear problem for the $\mathrm{KdV}$ equation, $\alpha=2$ in (1.3)) is bounded whenever $v_{0} \in H^{3 / 4}(\mathbb{R})$. Also in this case $(\alpha=2)$ the value $3 / 4$ appears in the estimate $(1.7)$, which is known to be sharp (i.e. (1.7), (1.8) do not hold if $s<(\alpha+1) / 4$, see [36]).

We will now give the precise statement of our results.

Theorem 1.1. (i) Let $s>3 / 4$. For any $u_{0} \in H^{s}(\mathbb{R})$ there exists a unique solution $u(t)$ of the IVP (1.1) satisfying

$$
u \in C\left([-T, T]: H^{s}\right)
$$

and

$$
\partial_{x} u \in L^{4}\left([-T, T]: L^{\infty}\right)
$$

Moreover,

$$
u \in L^{q}\left([-T, T]: L_{s+\theta \beta / 2}^{p}\right)
$$

for any $(\theta, \beta) \in[0,1] \times[0,1 / 2]$ with $(q, p)=(6 / \theta(\beta+1), 2 /(1-\theta))$,

$$
\sup _{x} \int_{-T}^{T}\left|D^{s} \partial_{x} u(x, t)\right|^{2} d t<\infty
$$

and

$$
\int \sup _{[-T, T]}\left|D^{r} u(x, t)\right|^{2} d x<\infty
$$

with $r<s-3 / 4$, and where $T$ has a lower bound depending only on $\left\|u_{0}\right\|_{s, 2}$.

(ii) For any $T^{\prime}<T$ there exists a neighborhood $V$ of $u_{0}$ in $H^{s}$ such that the map $\tilde{u}_{0} \rightarrow \widetilde{u}(t)$ from $V$ into the class defined by (1.11)-(1.15) with $T^{\prime}$ instead of $T$ is continuous.

(iii) If $u_{0} \in H^{s^{\prime}}$ with $s^{\prime}>s$, then (i)-(ii) hold with $s$ replaced by $s^{\prime}$ but with the same $T$.

(iv) For $\left\|u_{0}\right\|_{s, 2}$ sufficiently small

$$
T \geq c_{s}\left\|u_{0}\right\|_{s, 2}^{-1^{-}}
$$

where $a^{-}$denotes a fixed constant smaller than $a$. 
Theorem 1.2. If $s \geq 1$ the results in Theorem 1.1 are true with $T$ arbitrarily large.

Theorem 1.3. Let $\alpha \in[1,2)$ and $k=1$.

(i) For any $u_{0} \in H^{s}(\mathbb{R})$ with $s \geq(9-3 \alpha) / 4$ IVP (1.2) has a unique solution $u(t)$ satisfying (1.11)-(1.12). Moreover,

$$
u \in L^{q}\left([-T, T]: L_{s+\theta \beta / 2}^{p}\right)
$$

for any $(\theta, \beta) \in[0,1] \times[0,(\alpha-1) / 2]$ with

$$
\begin{aligned}
(q, p) & =(2(\alpha+1) / \theta(\beta+1), 2 /(1-\theta)), \\
u & \in L^{2}\left([-T, T]: H_{\mathrm{loc}}^{s+\alpha / 2}(\mathbb{R})\right),
\end{aligned}
$$

and

$$
\sum_{j=-\infty}^{\infty} \sup _{[j, j+1)} \sup _{[-T, T]}|u(x, t)|^{2}<\infty
$$

where $T$ has a lower bound depending only on $\left\|u_{0}\right\|_{s, 2}$.

(ii) For any $T^{\prime}<T$ there exists a neighborhood $V$ of $u_{0}$ in $H^{s}$ such that the map $\tilde{u}_{0} \rightarrow \widetilde{u}(t)$ from $V$ into the class defined by (1.11), (1.17)-(1.19) with $T^{\prime}$ instead of $T$ is continuous.

(iii) If $u_{0} \in H^{s^{\prime}}$ with $s^{\prime}>s$, then (i)-(ii) hold with $s$ replaced by $s^{\prime}$ but with the same $T$.

Theorem 1.4. If $s \geq \alpha / 2 \geq 9 / 10$ the results in Theorem 1.3 are true with $T$ arbitrarily large.

Some remarks are in order.

(a) Theorem 1.2 follows by combining Theorem 1.1 and the third conservation law $\Phi_{3}(\cdot)$. Similarly, Theorem 1.4 can be deduced from Theorem 1.3 by using the conservation law

$$
\varphi(u)=\int\left(\left(D^{\alpha / 2} u\right)^{2}-c_{k} u^{k+2}\right) d x
$$

for solutions of the IVP (1.2). Hence, their proof will be omitted.

(b) It will be clear from our proofs below that the results in Theorems 1.1 and 1.3 extend to any power $k \geq 2$ in (1.2) (the same applies to Theorems 1.2 and 1.4 with a possible restriction on the size of the data). However, in this case $(\alpha \in(1,2]$ and $k \geq 2$ in (1.2)) better results can be obtained. In particular, for the modified $\mathrm{KdV}$ equation, i.e., $(\alpha, k)=(2,2)$, it can be shown that the IVP (1.2) is locally well-posed in $H^{s}$ with $s \geq 1 / 4$. The proof in this case is different than those provided below for the case $k=1$. Therefore, it will be given somewhere else. Also we shall not consider here the IVP (1.2) with $\alpha>2$.

(c) Local well-posedness of the IVP (1.2) in $H^{s}$ was known for $s>3 / 2$ (see Saut [29]). Since $(9-3 \alpha) / 4 \leq 3 / 2$ for $\alpha \geq 1$, we obtain an improvement of 
this result. Notice that the number $(9-3 \alpha) / 4$ is equal to two minus $(\alpha-1) / 4$ (i.e., the gain of derivatives in the global smoothing (1.4)) minus $\alpha / 2$ (i.e., the gain of derivatives in the local smoothing (1.6)). We may also remark that in the limit $\alpha=1$ with $k=1$ global well-posedness has been established in $H^{s}$ with $s=3 / 2$ (see [27]).

(d) We have stated Theorem 1.1 in a form that emphasizes that the regularity of the solution $u(\cdot)$ of the nonlinear problem is exactly that of the associated linear problem $U^{2}(t) u_{0}$. Notice that in this case the form of the local smoothing effect (1.14) is stronger than all previously known forms (see (1.18) and the results in $[11,12,15,18])$.

(e) Theorem 1.1 (1.14), Theorem 1.3 (1.17), and the Sobolev embedding theorem show that although $u_{0}$ may be only Hölder continuous the solution $u(t)$ is $C^{1}$ a.e. in $t$.

(f) The estimate (1.16) for the lower bound of the life span of small solutions of (1.1) is similar to that deduced in [30, Proposition 2.1] for the case $s>3 / 2$.

(g) Finally, it should be mentioned that with the ingredients discussed in this introduction a proof of Theorem 1.1 based on the contraction principle (with respect to the norms in (1.11)-(1.15)) can be easily obtained. For this, one only needs the integral equation

$$
u(t)=U^{2}(t) u_{0} \int_{0}^{t} U^{2}(t-\tau) u \partial_{x} u(\tau) d \tau
$$

the estimates (1.4), (1.6), and (1.7), and the commutator estimates in Lemma 2.10. In particular, it does not use the energy estimate (1.10). The so-called loss of derivatives obtained from estimates involving the integral equation is overcome by combining the estimates (1.6) and (1.7) as in (4.14)-(4.16) below. However, this approach does not seem to work when $\alpha<2$. Thus we prefer to present a method which applies to any $\alpha \in[1,2]$.

This paper is organized as follows. In $\S 2$, we prove all the linear estimates discussed in this introduction. In $\S 3$, we explain the common part in the method of proof of Theorems 1.1 and 1.3. Sections 4 and 5 are concerned with the proof of Theorems 1.1 and 1.3 respectively.

\section{Notation.}

- The norm in $L^{p}(\mathbb{R}), 1 \leq p \leq \infty$, will be denoted by $\|\cdot\|_{p}$.

- $J^{s}=(1-\Delta)^{s / 2}$ and $D^{s}=(-\Delta)^{s / 2}$ denote the Bessel and Riesz potential of order $-s$ respectively.

- $\sigma$ is the Hilbert transform, i.e., $\widehat{\sigma f}=i \operatorname{sgn}(\xi) \widehat{f}$. In particular, $\partial_{x} \circ \sigma$ $=D$.

- $L_{s}^{p}=J^{-s} L^{p}$, whose norm will be denoted by $\|\cdot\|_{s, p}=\left\|J^{s} \cdot\right\|_{p}$. When $p=2$ we will write $H^{s}$ instead of $L_{s}^{2} . H^{\infty}=\bigcap_{s>0} H^{s}$.

- $H_{\text {loc }}^{s}(\mathbb{R}):$ for $s>0, f \in L_{\text {loc }}^{2}(\mathbb{R})$ such that for every $\varphi \in C_{0}^{\infty}(\mathbb{R})$, $\varphi f \in H^{S}(\mathbb{R})$.

- $S(\mathbb{R})$ denotes the Schwartz class. 
- $[A ; B] \equiv A B-B A$, where $A, B$ are operators. Thus, $\left[J^{s} ; f\right] g=$ $J^{s}(f g)-f J^{s} g$ in which $f$ is regarded as a multiplication operator.

- $\chi: \mathbb{R} \rightarrow \mathbb{R}$ denotes a nondecreasing $C^{\infty}$-function such that $\chi^{\prime}=1$ on $[0,1]$ and $\operatorname{supp} \chi^{\prime} \subseteq(-1,2)$. For any $j \in Z, \chi_{j}(\cdot)=\chi(\cdot-j)$.

- If $a$ is any real number, $a^{+}\left(a^{-}\right)$denotes any number bigger (smaller) than $a$.

\section{LINEAR ESTIMATES}

In the first part of this section we shall deduce the estimates (1.4), (1.6)(1.8) described in the introduction. The second part is concerned with some commutator estimates needed in the proofs of Theorems 1.1 and 1.3.

The solution of the linear IVP

$$
\left\{\begin{array}{l}
\partial_{t} v-D^{\alpha} \partial_{x} v=0, \quad t, x \in \mathbb{R}, \alpha \geq 1 \\
v(x, 0)=v_{0}(x)
\end{array}\right.
$$

is given by the unitary group $\left\{U^{\alpha}(t)\right\}_{-\infty}^{\infty}$, i.e., $v(\cdot, t)=U^{\alpha}(t) v_{0}=S_{t}^{\alpha} * v_{0}$, where for $t>0$

$$
S_{t}^{\alpha}(\cdot)=t^{-1 /(\alpha+1)} K^{\alpha}\left(t^{-1 /(\alpha+1)} \cdot\right)
$$

and

$$
K^{\alpha}(x)=c \int_{-\infty}^{\infty} e^{i\left(\xi|\xi|^{\alpha}+x \xi\right)} d \xi
$$

We begin by proving the sharp version (1.6) of the local smoothing effect in solutions of (2.1).

Lemma 2.1. Let $v_{0} \in L^{2}(\mathbb{R})$. Then

$$
\int_{-\infty}^{\infty}\left|D^{\alpha / 2} U^{\alpha}(t) v_{0}(x)\right|^{2} d t=c_{\alpha}\left\|v_{0}\right\|_{2}^{2}
$$

for any $x \in \mathbb{R}$.

Proof. Performing the change of variable $\eta=\xi|\xi|^{\alpha}$, using Plancherel's Theorem in the $t$-variable, and then returning to the original variable $\xi=\phi(\eta)$, it follows that

$$
\begin{aligned}
\int\left|D^{\alpha / 2} U^{\alpha}(t) v_{0}\right|^{2} d t & =\left.\left.\int\left|\int e^{\left.i t \xi \xi \xi\right|^{\alpha}} e^{i x \xi}\right| \xi\right|^{\alpha / 2} \widehat{v}_{0}(\xi) d \xi\right|^{2} d t \\
& =\left.\left.\int\left|\int e^{i t \eta} e^{i x \phi(\eta)}\right| \phi(\eta)\right|^{\alpha / 2} \widehat{v}_{0}(\phi(\eta)) \phi^{\prime}(\eta) d \eta\right|^{2} d t \\
& =\left.\left.c \int\left|e^{i x \phi(\eta)}\right| \phi(\eta)\right|^{\alpha / 2} \widehat{v}_{0}(\phi(\eta)) \phi^{\prime}(\eta)\right|^{2} d \eta \\
& =c_{\alpha} \int\left|\widehat{v}_{0}(\xi)\right|^{2} d \xi .
\end{aligned}
$$

Next we shall sketch the proof of the global smoothing effect (1.4). This is based on the following estimates for the time behavior of the derivatives of order $\gamma \in[0,(\alpha-1) / 2]$ of the kernel $S_{t}^{\alpha}(\cdot)$. 
Lemma 2.2. If $\gamma \in[0,(\alpha-1) / 2]$, and

then

$$
D^{\gamma} S_{t}^{\alpha}(x)=c \int e^{i\left(t \xi|\xi|^{\alpha}+x \xi\right)}|\xi|^{\gamma} d \xi
$$

$$
\left\|D^{\gamma} S_{t}^{\alpha}(\cdot)\right\|_{\infty} \leq c|t|^{-(\gamma+1) /(\alpha+1)} .
$$

Corollary 2.3. For any $(\theta, \beta) \in[0,1] \times[0,(\alpha-1) / 2]$

$$
\left\|D^{\theta \beta} S_{t} * v_{0}\right\|_{2 /(1-\theta)} \leq C|t|^{-\theta(\beta+1) /(\alpha+1)}\left\|v_{0}\right\|_{2 /(1+\theta)} .
$$

For the proofs of (2.3) and (2.4) we refer to [18, Proposition 2.3; 28, Corollary 2.3].

Theorem 2.4. For any $(\theta, \beta) \in[0,1] \times[0,(\alpha-1) / 2]$

$$
\left(\int_{-\infty}^{\infty}\left\|D^{\theta \beta / 2} U^{\alpha}(t) v_{0}\right\|_{p}^{q} d t\right)^{1 / q} \leq c\left\|v_{0}\right\|_{2}
$$

and

$$
\left(\int_{-\infty}^{\infty}\left\|\int D^{\theta \beta} U^{\alpha}(t-\tau) f(\cdot, \tau) d \tau\right\|_{p}^{q} d t\right)^{1 / q} \leq c\left(\int_{-\infty}^{\infty}\|f(\cdot, t)\|_{p^{\prime}}^{q^{\prime}} d t\right)^{1 / q^{\prime}},
$$

where $(q, p)=(2(\alpha+1) / \theta(\beta+1), 2 /(1-\theta))$ and $\frac{1}{p}+\frac{1}{p^{\prime}}=\frac{1}{q}+\frac{1}{q^{\prime}}=1$.

Proof. We shall follow the method used in $[10,23,26,35]$.

By duality it is easy to see that $(2.5)$ is equivalent to

$$
\left\|\int D^{\theta \beta / 2} U^{\alpha}(t) f(\cdot, t) d t\right\|_{2} \leq c\left(\int\|f(\cdot, t)\|_{p^{\prime}}^{q^{\prime}} d t\right)^{1 / q^{\prime}} .
$$

On the other hand, following P. Tomas' argument in [35] we find that

$$
\begin{gathered}
\int\left(\int D^{\theta \beta / 2} U^{\alpha}(t) f(\cdot, t) d t \cdot \int D^{\theta \beta / 2} \overline{U^{\alpha}(\tau) g(\cdot, \tau)} d \tau\right) d x \\
=\iint f(x, t)\left(\int D^{\theta \beta} U^{\alpha}(t-\tau) \overline{g(\cdot, \tau)} d \tau\right) d x d t .
\end{gathered}
$$

Hence, (2.5), (2.6), and (2.7) are equivalent.

To prove $(2.6)$, as in $[10,23,26]$ one uses Minkowski's integral inequality, the decay in time estimate (2.4), and fractional integration to obtain that the left-hand side of $(2.6)$ is bounded by

$$
\begin{aligned}
& \left\|\int\right\| D^{\theta \beta} U^{\alpha}(t-\tau) g(\cdot, \tau)\left\|_{p} d \tau\right\|_{q} \\
& \quad \leq c\left\|\int|t-\tau|^{-\theta(\beta+1) /(\alpha+1)}\right\| g(\cdot, \tau)\left\|_{p^{\prime}} d \tau\right\|_{q} \\
& \quad \leq c\left(\int\|g(\cdot, t)\|_{p^{\prime}}^{q^{\prime}} d t\right)^{1 / q^{\prime}},
\end{aligned}
$$

where $\frac{1}{q}=1-\frac{1}{q^{\prime}}=\frac{1}{q^{\prime}}-\left(1-\frac{\theta(\beta+1)}{\alpha+1}\right)$, i.e., $q=2(\alpha+1) / \theta(\beta+1)$. 
To establish the estimates (1.7) and (1.8) we need the following version of the classical Van der Corput lemma.

Lemma 2.5. Let $\psi \in C_{0}^{\infty}(\mathbb{R})$ and $\phi \in C^{2}(\mathbb{R})$ satisfy that $\phi^{\prime \prime}(\xi)>\lambda>0$ on the support of $\psi$. Then

$$
\left|\int e^{i \phi(\xi)} \psi(\xi) d \xi\right| \leq 10 \lambda^{-1 / 2}\left\{\|\psi\|_{\infty}+\left\|\psi^{\prime}\right\|_{1}\right\}
$$

Proof. See [32, pp. 309-311].

As a consequence of this lemma we have the following result.

Proposition 2.6. Let $\psi$ be a $C^{\infty}$ function supported in the interval $\left[2^{k-1}, 2^{k+1}\right]$ where $k$ is a natural number. For $\alpha \geq 1$ the function $H_{k}^{\alpha}(\cdot)$, defined as

$$
H_{k}^{\alpha}(x)= \begin{cases}2^{k} & \text { if }|x| \leq 1, \\ 2^{k / 2}|x|^{-1 / 2} & \text { if } 1 \leq|x| \leq c 2^{\alpha k}, \\ 1 /\left(1+x^{2}\right) & \text { if }|x|>c 2^{\alpha k},\end{cases}
$$

satisfies

$$
\left|\int_{-\infty}^{\infty} e^{i\left(\left.t \xi \xi \xi\right|^{\alpha}+x \xi\right)} \psi(\xi) d \xi\right| \leq c H_{k}^{\alpha}(x)
$$

for $|t| \leq 2$, where $c$ does not depend on $t$ or $k$.

Proof. To simplify the exposition we assume $t \in[0,2]$. Define $\Omega=\{\xi \in$ supp $\left.\psi|| \alpha t|\xi|^{\alpha}+x|\leq| x \mid / 2\right\}$ and choose $\varphi$ in $C^{\infty}$ supported in $\Omega$ such that $\varphi \equiv 1$ when $\left.|\alpha t| \xi\right|^{\alpha}+x|\leq| x \mid / 3$.

If $\xi \in \Omega$ then $|x| \sim \alpha t|\xi|^{\alpha} \leq c 2^{\alpha k}$. In this case the phase function $\phi_{x}(\xi)=$ $t \xi|\xi|^{\alpha}+x \xi$ satisfies

$$
\left|\phi^{\prime \prime}(\xi)\right|=c t|\xi|^{\alpha-1} \geq c 2^{-k}|x| .
$$

Thus, from Lemma 2.5 we find that

$$
\left|\int e^{i \phi_{x}(\xi)}(\varphi \psi)(\xi) d \xi\right| \leq c 2^{k / 2}|x|^{-1 / 2} .
$$

If $\xi \in \operatorname{supp}(1-\varphi)$, then $\left|\phi_{x}^{\prime}(\xi)\right|=\left.|\alpha t| \xi\right|^{\alpha}+x|\geq| x \mid / 3$. Hence, using integration by parts it follows that for $|x| \geq 1$

$$
\left|\int e^{i \phi_{x}(\xi)}((1-\varphi) \psi)(\xi) d \xi\right| \leq \frac{c}{1+x^{2}} .
$$

Combining these estimates one obtains the desired result. 
Theorem 2.7. For any $s>(\alpha+1) / 4$ with $\alpha \geq 1$

$$
\left(\sum_{j=-\infty}^{\infty} \sup _{|t| \leq 1} \sup _{j \leq x<j+1}\left|U^{\alpha}(t) v_{0}(x)\right|^{2}\right)^{1 / 2} \leq c\left\|v_{0}\right\|_{s, 2} .
$$

Proof. Let $\left\{\psi_{k}\right\}_{k=0}^{\infty}$ be a smooth partition of unity of $\mathbb{R}^{+}$such that

$$
\operatorname{supp} \psi_{k} \subseteq\left[2^{k-1}, 2^{k+1}\right] \text { for } k=1,2, \ldots,
$$

and $\operatorname{supp} \psi_{0} \subseteq[-1,1]$. Define $\left(U_{k}^{\alpha}(t) v_{0}\right)^{\wedge}(\xi)=e^{i t \xi|\xi|^{\alpha}} \psi_{k}(|\xi|) \hat{v}_{0}(\xi)$. Notice that since $|t| \leq 1$ the support of $\left(U_{k}^{\alpha}(t) v_{0}\right)^{\wedge}(\xi)$ is contained in $\left\{\xi /|\xi| \leq 2^{k+1}\right\}$. Thus it suffices to show that

$$
\left(\sum_{j=-\infty}^{\infty} \sup _{|t| \leq 1} \sup _{j \leq x<j+1}\left|U_{k}^{\alpha}(t) v_{0}(x)\right|^{2}\right)^{1 / 2} \leq c 2^{(\alpha+1) k / 4}\left\|v_{0}\right\|_{2}
$$

Using a duality argument, it suffices to prove that (2.9)

$$
\left\|\int_{-1}^{1} U_{k}^{\alpha}(t) g(\cdot, t) d t\right\|_{2} \leq c 2^{(\alpha+1) k / 4}\left(\sum_{j}\left(\int_{-1}^{1} \int_{j}^{j+1}|g(x, t)| d x d t\right)^{2}\right)
$$

and by $\mathrm{P}$. Tomas' argument [35] one easily sees (as in the proof of Theorem 2.4) that (2.9) is a consequence of the following estimate :

$$
\begin{aligned}
& \left(\sum_{j=-\infty}^{\infty} \sup _{|t| \leq 1} \sup _{j \leq x<j+1}\left|\int_{-1}^{1} U_{k}^{\alpha}(t-\tau) g(\cdot, \tau) d \tau\right|^{2}\right)^{1 / 2} \\
& \leq c 2^{(\alpha+1) k / 2}\left(\sum_{j=-\infty}^{\infty}\left(\int_{-1}^{1} \int_{j}^{j+1}|g(x, t)| d x d t\right)^{2}\right)^{1 / 2} .
\end{aligned}
$$

To establish (2.10) we use Proposition 2.6 to find that

$$
\begin{aligned}
& \left|\left(\int_{-1}^{1} U_{k}^{\alpha}(t-\tau) g(\cdot, \tau) d \tau\right)(x)\right| \\
& \quad \leq \int H_{k}^{\alpha}(y) \int_{-1}^{1}|g(x-y, \tau)| d \tau d y \\
& \quad \leq \sum_{l=-\infty}^{\infty} H_{k}^{\alpha}(|l|) \int_{l}^{l+1} \int_{-1}^{1}|g(x-y, \tau)| d \tau d y .
\end{aligned}
$$


Hence, the left-hand side of $(2.10)$ is bounded by

$$
\begin{aligned}
& \left(\sum_{j=-\infty}^{\infty}\left(\sup _{j \leq x<j+1} \sum_{l=-\infty}^{\infty} H_{k}^{\alpha}(|l|) \int_{l}^{l+1} \int_{-1}^{1}|g(x-y, \tau)| d \tau d y\right)^{2}\right)^{1 / 2} \\
& \leq\left(\sum_{j=-\infty}^{\infty}\left(\sum_{l=-\infty}^{\infty} H_{k}^{\alpha}(|l|) \int_{l-j-1}^{l-j+2} \int_{-1}^{1}|g(z, \tau)| d \tau d z\right)^{2}\right)^{1 / 2} \\
& \leq \sum_{l=-\infty}^{\infty} H_{k}^{\alpha}(|l|)\left(\sum_{j=-\infty}^{\infty}\left(\int_{l-j+1}^{l-j+2} \int_{-1}^{1}|g(z, \tau)| d \tau d z\right)^{2}\right)^{1 / 2} \\
& \leq 4 \sum_{l=-\infty}^{\infty} H_{k}^{\alpha}(|l|)\left(\sum_{j=-\infty}^{\infty}\left(\int_{j}^{j+1} \int_{-1}^{1}|g(x, t)| d t d x\right)^{2}\right)^{1 / 2} \\
& \leq c 2^{(\alpha+1) k / 2}\left(\sum_{j=-\infty}^{\infty}\left(\int_{-1}^{1} \int_{j}^{j+1}|g(x, t)| d x d t\right)^{2}\right)^{1 / 2}
\end{aligned}
$$

which agrees with the right hand side of (2.10).

As consequences of Theorem 2.7 we obtain the estimates (1.7) and (1.8) described in the introduction.

Corollary 2.8. For any $s>(\alpha+1) / 4$ and any $\rho>3 / 4$

$$
\left(\sum_{j=-\infty}^{\infty} \sup _{|t| \leq T} \sup _{j \leq x<j+1}\left|U^{\alpha}(t) v_{0}(x)\right|^{2}\right)^{1 / 2} \leq c(1+T)^{\rho}\left\|v_{0}\right\|_{s, 2}
$$

Proof. Denote $t^{\prime}=T t$ with $|t| \leq 1$. An easy calculation shows that

$$
U^{\alpha}\left(t^{\prime}\right) v_{0}(x)=U^{\alpha}(t) v_{1}\left(\frac{x}{T^{1 /(1+\alpha)}}\right)
$$

where $\widehat{v}_{1}(\xi)=T^{-1 /(1+\alpha)} \widehat{v}_{0}\left(T^{-1 /(1+\alpha)} \xi\right)$. Therefore, we see that

$$
\begin{aligned}
& \sum_{j=-\infty}^{\infty} \sup _{\left|t^{\prime}\right| \leq T} \sup _{j \leq x<j+1}\left|U^{\alpha}\left(t^{\prime}\right) v_{0}(x)\right|^{2} \\
& \quad=\sum_{j=-\infty}^{\infty} \sup _{|t| \leq 1} \sup _{j \leq x \cdot T^{1 /(\alpha+1)}<j+1}\left|U^{\alpha}(t) v_{1}(x)\right|^{2} \\
& \quad \leq c T^{1 /(\alpha+1)} \sum_{k=-\infty}^{\infty} \sup _{|t| \leq 1} \sup _{k \leq x<k+1}\left|U^{\alpha}(t) v_{1}(x)\right|^{2} .
\end{aligned}
$$

From (2.8) the above expression is bounded by

$$
T^{1 /(\alpha+1)}\left\|v_{1}\right\|_{s, 2} \leq T^{s /(1+\alpha)}\left\|v_{0}\right\|_{s, 2} \text {. }
$$

Now take $r>1 / 4$ such that $r(1+\alpha)=s_{0}>\frac{1+\alpha}{4}$. If $s<s_{0}$ we have obtained the result. If $s_{0} \leq s$ we have proved that (2.11) is majorized by $T^{r}\left\|v_{0}\right\|_{s_{0}, 2} \leq$ $T^{r}\left\|v_{0}\right\|_{s, 2}$. 
Corollary 2.9. For any $s>(\alpha+1) / 4$ and any $\rho>3 / 4$

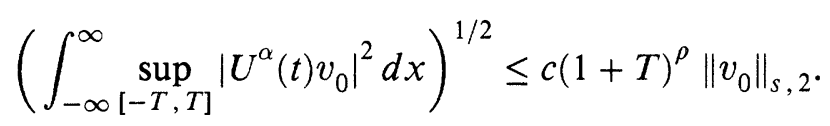

Proof. As was mentioned in the introduction, (2.12) follows directly from (2.11).

The last corollary is due to Vega [37]. The following version of (2.12) was essentially proved by Kenig and Ruiz in [20] for $\alpha \geq 1$ :

$$
\left(\int_{-\infty}^{\infty} \sup _{[-1,1]}\left|U^{\alpha}(t) v_{0}\right|^{4} d x\right)^{1 / 4} \leq c\left\|D^{1 / 4} v_{0}\right\|_{2} .
$$

To complete this section we shall state the commutator estimates needed in the coming sections.

Thus we have the estimates established by Kato and Ponce [17] as applications of the Coifman and Meyer results in [7].

Lemma 2.10. Let $s>0$ and $1<p<\infty$. If $f, g \in S\left(\mathbb{R}^{n}\right)$, then

$$
\left\|J^{s}(f g)-f J^{s} g\right\|_{p} \leq c\left\{\|\nabla f\|_{p_{1}}\|g\|_{s-1, p_{2}}+\|f\|_{s, p_{3}}\|g\|_{p_{4}}\right\}
$$

and

$$
\left\|J^{s}(f g)\right\|_{p} \leq c\left\{\|f\|_{p_{1}}\|g\|_{s, p_{2}}+\|f\|_{s, p_{3}}\|g\|_{p_{4}}\right\}
$$

with $p_{2}, p_{3} \in(1, \infty)$ such that $\frac{1}{p}=\frac{1}{p_{1}}+\frac{1}{p_{2}}=\frac{1}{p_{3}}+\frac{1}{p_{4}}$.

It is clear that $(2.13)-(2.14)$ are true whenever the corresponding right-hand side is finite.

Proof. The proof of this lemma is essentially contained in the one given in [17, Appendix] for the case $p_{1}=p_{4}=\infty$. One just needs to combine the argument used in [17] with the version of the R. R. Coifman and Y. Meyer result found in [7, pp. 22].

\section{THE NONLINEAR PROBLEM}

This section is concerned with general properties of solutions of the nonlinear IVP

$$
\left\{\begin{array}{l}
\partial_{t} u-D^{\alpha} \partial_{x} u+u \partial_{x} u=0, \quad x, t \in \mathbb{R}, \\
u(x, 0)=u_{0}(x),
\end{array}\right.
$$

with $\alpha \in[1,2]$. The properties collected here all appear in the previous literature.

We begin by stating the following local existence theorem proved in $[14,30]$. 
Theorem 3.1. (i) For any $u_{0} \in H^{s}(\mathbb{R})$ with $s>3 / 2$ there exists a unique solution $u(\cdot)$ to $(3.1)$ in the class

$$
C\left([-T, T]: H^{s}(\mathbb{R})\right)
$$

with $T=T\left(\left\|u_{0}\right\|_{s, 2}\right)>0$.

(ii) For any $T^{\prime}<T$ there exists a neighborhood $V$ of $u_{0}$ in $H^{s}(\mathbb{R})$ such that the map $\widetilde{u}_{0} \rightarrow \widetilde{u}(t)$ from $V$ into $C\left(\left[-T^{\prime}, T^{\prime}\right]: H^{s}(\mathbb{R})\right)$ is continuous.

(iii) If $u_{0} \in H^{s^{\prime}}(\mathbb{R})$ with $s^{\prime}>s$, then the time of existence $T$ can be taken to depend only on $\left\|u_{0}\right\|_{s, 2}$.

Next, for $u_{0} \in L^{2}(\mathbb{R})$ and $\varepsilon>0$, we consider the IVP

$$
\left\{\begin{array}{l}
\partial_{t} u-D^{\alpha} \partial_{x} u+u \partial_{x} u=0, \quad x, t \in \mathbb{R}, \alpha \in[1,2], \\
u(x, 0)=\varphi_{\varepsilon} * u_{0}=u_{0}^{\varepsilon},
\end{array}\right.
$$

where $\varphi \in S(\mathbb{R})$ with $\int \varphi(x) d x=1, \int x^{k} \varphi(x) d x=0, k=1,2, \ldots$, and $\varphi_{\varepsilon}(x)=\varepsilon^{-1} \varphi(x / \varepsilon)$.

From Theorem 3.1 and its proof, it follows that for general $u_{0} \in L^{2}(\mathbb{R})$ the IVP (3.2) has a unique solution $u^{\varepsilon}(\cdot)$ such that

$$
u^{\varepsilon} \in\left(\left[-T_{\varepsilon}, T_{\varepsilon}\right]: H^{\infty}(\mathbb{R})\right)
$$

with $T_{\varepsilon} \rightarrow 0$ as $\varepsilon \rightarrow 0$.

Our first goal will be to obtain an a priori estimate for the life-span interval $[-T, T]$ of the solution $u^{\varepsilon}(\cdot)$ of (3.2) with $T$ independent of $\varepsilon$ whenever $u_{0} \in H^{s}(\mathbb{R})$ with $s>3 / 4$ if $\alpha=2$, and $s \geq(9-3 \alpha) / 4$ if $\alpha \in[1,2)$.

To achieve this goal we need the following two lemmas. The first one is concerned with energy estimates for solutions of the IVP (3.1). The second is a simple continuation principle for solutions of the IVP (3.1).

Lemma 3.2. Let $u \in C\left([-T, T]: H^{\infty}\right)$ be a solution of the IVP (3.1). Then for any $s>0$

$$
\sup _{[-T, T]}\|u(t)\|_{s, 2} \leq c_{s}\left\|u_{0}\right\|_{s, 2} \exp \left(\int_{-T}^{T}\left\|\partial_{x} u(\tau)\right\|_{\infty} d \tau\right) .
$$

Proof. Applying the operator $J^{s}$ to the equation in (3.1) leads to the identity

$$
\partial_{t} J^{s} u-D^{\alpha} \partial_{x} J^{s} u+u \partial_{x} J^{s} u+\left[J^{s} ; u\right] \partial_{x} u=0 \text {. }
$$

Using integration by parts and the estimate (2.14) we find that

$$
\frac{d}{d t}\|u(t)\|_{s, 2}^{2}=\int \partial_{x} u\left(J^{s} u\right)^{2}-2 \int\left[J^{s} ; u\right] \partial_{x} u J^{s} u \leq c\left\|\partial_{x} u\right\|_{\infty}\|u(t)\|_{s, 2}^{2} .
$$

Thus Gronwall's inequality yields (3.3).

Lemma 3.3. If $u \in C\left([0, T]: H^{\infty}\right)$ is a solution of the IVP (3.1), and for some $T_{1}>T$

$$
\int_{0}^{T_{1}}\left\|\partial_{x} u(t)\right\|_{\infty} d t=M<\infty
$$


then the solution $u(\cdot)$ can be extended to a solution of the same class in the time interval $\left[0, T_{1}\right]$.

Proof. By inserting (3.4) in (3.3) it follows that

$$
\sup _{[0, T]}\|u(t)\|_{s, 2} \leq c_{s}\left\|u_{0}\right\|_{s, 2} \exp (M)
$$

for any $s>0$. Since the right-hand side of (3.5) is independent of $t \in\left[0, T_{1}\right]$, by reapplying Theorem 3.1 one obtains the desired result.

The goal discussed at the end of Theorem 3.1 reduces to establishing the estimate (3.4) for solutions of the IVP (3.2) with a constant $M$ independent of $\varepsilon$. This will be proved in two slightly different manners, for the cases $\alpha=2$ (Theorem 1.1) and $\alpha \in[1,2$ ) (Theorem 1.3).

\section{Proof of Theorem 1.1}

Combining the estimates (1.4), (1.6), and (1.7) for the group $\left\{U^{\alpha}(t)\right\}_{-\infty}^{\infty}$, proved in $\S 2$, with the results in the previous section we obtain the key step in the proof of this theorem.

Lemma 4.1. For any $u_{0} \in H^{s}(\mathbb{R})$ with $s>3 / 4$ there exists $T>0$ such that if $u=u^{\varepsilon} \in C\left([-T, T]: H^{\infty}(\mathbb{R})\right)$ is a solution of the IVP (3.2) with $\alpha=2$ and $\varepsilon \in(0,1)$, then

$$
\begin{gathered}
\sup _{[-T, T]}\|u(t)\|_{s, 2} \leq K, \\
\left(\int_{-T}^{T}\left\|J^{l} \partial_{x} u(t)\right\|_{\infty}^{4} d t\right)^{1 / 4} \leq K, \\
\sup _{x}\left(\int_{-T}^{T}\left|D^{s} \partial_{x} u(x, t)\right|^{2} d t\right)^{1 / 2} \leq K,
\end{gathered}
$$

and

$$
\left(\int \sup _{[-T, T]}\left|J^{r} u(x, t)\right|^{2} d x\right)^{1 / 2} \leq K
$$

for any $(l, r) \in[0, s-3 / 4] \times[0, s-3 / 4)$, and where $K$ depends only on $\left\|u_{0}\right\|_{s, 2}$. In fact, for $\left\|u_{0}\right\|_{s, 2}$ sufficiently small

$$
T \geq c_{s}\left\|u_{0}\right\|_{s, 2}^{-1^{-}}
$$

Furthermore, if $u_{0} \in H^{s^{\prime}}(\mathbb{R})$ with $s^{\prime}>s$, then (4.1)-(4.4) hold with $s^{\prime}$ instead of $s$ (and $K^{\prime}=K^{\prime}\left(\left\|u_{0}\right\|_{s^{\prime}, 2}\right)$ instead of $\left.K\right)$ in the same interval $[-T, T]$.

Proof. To simplify the exposition we restrict ourselves to the case $t \geq 0$, and introduce the following notation:

$$
\gamma(T, s)=\sup _{[0, T]}\|u(t)\|_{s, 2}
$$




$$
\begin{gathered}
\lambda(T)=\left(\int_{0}^{T}\left\|\partial_{x} u(t)\right\|_{\infty}^{4} d t\right)^{1 / 4} \\
\sigma(T, s)=\sup _{x}\left(\int_{0}^{T}\left|D^{s} \partial_{x} u(x, t)\right|^{2} d t\right)^{1 / 2}
\end{gathered}
$$

and

$$
\nu(T)=\left(\int \sup _{[0, T]}|u(x, t)|^{2} d x\right)^{1 / 2} .
$$

Thus, using Hölder's inequality, the energy estimate (3.3) can be written as

$$
\gamma(T, s) \leq c_{s}\left\|u_{0}\right\|_{s, 2} \exp \left(T^{3 / 4} \lambda(T)\right)
$$

Next, from the integral equation

$$
u(t)=U^{2}(t) u_{0}-\int_{0}^{t} U^{2}(t-\tau)\left(u \partial_{x} u(\tau)\right) d \tau
$$

we find that

$$
\partial_{x} u(t)=D^{1 / 4} U^{2}(t) D^{3 / 4} \sigma u_{0}-\int_{0}^{t} D^{1 / 4} U^{2}(t-\tau) D^{3 / 4} \sigma\left(u \partial_{x} u\right)(\tau) d \tau,
$$

where $\sigma$ denotes the Hilbert transform.

Hence, using (2.5) with $(\alpha, \theta, \beta)=(2,1,1 / 2)$ in (4.12) it follows that

$$
\lambda(T) \leq c\left\|u_{0}\right\|_{3 / 4,2}+c \int_{0}^{T}\left\|D^{3 / 4}\left(u \partial_{x} u\right)(\tau)\right\|_{2} d \tau .
$$

Similarly, using (2.2) and (2.12) in (4.11) we find that

$$
\sigma(T, s) \leq c\left\|u_{0}\right\|_{s, 2}+c \int_{0}^{T}\left\|D^{s}\left(u \partial_{x} u\right)(\tau)\right\|_{2} d \tau
$$

and

$$
\nu(T) \leq c(1+T)^{1 / 4^{+}}\left\{\left\|u_{0}\right\|_{s, 2}+\int_{0}^{T}\left\|J^{3 / 4^{+}}\left(u \partial_{x} u\right)(\tau)\right\|_{2} d \tau\right\} .
$$

Thus, we should estimate the integral terms in (4.13)-(4.15). First, using Mihlin's theorem we observe that

$$
\begin{aligned}
\left\|J^{s}\left(u \partial_{x} u\right)\right\|_{2} & =\left\|u D^{s} \partial_{x} u+u\left(J^{s}-D^{s}\right) \partial_{x} u+\left[J^{s} ; u\right] \partial_{x} u\right\| \\
& \leq\left\|u D^{s} \partial_{x} u\right\|_{2}+\|u\|_{\infty}\|u\|_{s, 2}+\left\|\left[J^{s} ; u\right] \partial_{x} u\right\|_{2}
\end{aligned}
$$

Then, by combining (2.13), (2.14), the Cauchy-Schwarz and Hölder inequalities, the Sobolev embedding theorem, and (4.10), it is not hard to obtain the 
following string of inequalities:

(4.16)

$$
\begin{aligned}
& \int_{0}^{T}\left\|J^{s}\left(u \partial_{x} u\right)(t)\right\|_{2} d t \\
& \leq c \int_{0}^{T}\left(\left\|u D^{s} \partial_{x} u\right\|_{2}+\left\|\left[J^{s} ; u\right] \partial_{x} u\right\|_{2}+\|u\|_{\infty}\|u\|_{s, 2}\right)(t) d t \\
& \leq c T^{1 / 2}\left(\int_{0}^{T} \int\left|u D^{s} \partial_{x} u\right|^{2} d x d t\right)^{1 / 2} \\
& +c \int_{0}^{T}\left(\left\|\partial_{x} u\right\|_{\infty}+\|u\|_{\infty}\right)\|u\|_{s, 2}(t) d t \\
& \leq c T^{1 / 2}\left(\iint_{0}^{T}\left|u D^{s} \partial_{x} u\right|^{2} d t d x\right)^{1 / 2}+c T^{3 / 4}\left(\int_{0}^{T}\left\|\partial_{x} u(t)\right\|_{\infty}^{4} d t\right)^{1 / 4} \\
& \times \sup _{[0, T]}\|u(t)\|_{s, 2}+c T \sup _{[0, T]}\|u(t)\|_{3 / 4,2} \sup _{[0, T]}\|u(t)\|_{s, 2} \\
& \leq c T^{1 / 2}\left(\int \sup _{[0, T]}|u(x, t)|^{2} d x\right)^{1 / 2}\left(\sup _{x} \int_{0}^{T}\left|D^{s} \partial_{x} u(x, t)\right|^{2} d t\right)^{1 / 2} \\
& +c T^{3 / 4} \lambda(T)\left\|u_{0}\right\|_{s, 2} \exp \left(T^{3 / 4} \lambda(T)\right) \\
& +c T\left\|u_{0}\right\|_{3 / 4,2}\left\|u_{0}\right\|_{s, 2} \exp \left(2 T^{3 / 4} \lambda(T)\right) \\
& \leq c T^{1 / 2} \nu(T) \sigma(T, s)+c T^{3 / 4}\left\|u_{0}\right\|_{s, 2} \lambda(T) \exp \left(T^{3 / 4} \lambda(T)\right) \\
& +c T\left\|u_{0}\right\|_{3 / 4,2}\left\|u_{0}\right\|_{s, 2} \exp \left(2 T^{3 / 4} \lambda(T)\right) .
\end{aligned}
$$

Inserting (4.16) in (4.13)-(4.15) we see that

$$
\begin{aligned}
\max & \left\{\lambda(T) ; \sigma(T, s) ;(1+T)^{-1 / 4^{+}} \nu(T)\right\} \\
\leq & c\left\|u_{0}\right\|_{s, 2}+c T^{1 / 2} \nu(T) \sigma(T, s) \\
& +c T^{3 / 4}\left\|u_{0}\right\|_{s, 2} \exp \left(2 T^{3 / 4} \lambda(T)\right)\left(\lambda(T)+T^{1 / 4}\left\|u_{0}\right\|_{3 / 4,2}\right) .
\end{aligned}
$$

Notice that $\gamma(\cdot, s), \lambda(\cdot), \sigma(\cdot, s)$, and $\nu(\cdot)$ are continuous nondecreasing functions and that the terms in the right-hand side of (4.17) depend linearly on the norms involving derivatives of order $s$. Hence, defining $T_{0}$ by the expression

$$
\begin{aligned}
& \max \left\{T_{0}^{3 / 4} \lambda\left(T_{0}\right) ; c T_{0}^{1 / 2}\left(1+T_{0}\right)^{1 / 4^{+}} \sigma\left(T_{0}, s\right)\right. \\
& \left.\quad c T_{0}^{1 / 2}\left(1+T_{0}\right)^{1 / 4^{+}} \nu\left(T_{0}\right) ; T_{0}\left\|u_{0}\right\|_{3 / 4,2}\right\}=1 / 2
\end{aligned}
$$

from (4.17) we can conclude that

$$
\max \left\{\lambda\left(T_{0}\right) ; \sigma\left(T_{0}, s\right) ;\left(1+T_{0}\right)^{-1 / 4^{+}} \nu\left(T_{0}\right)\right\} \leq c\left\|u_{0}\right\|_{s, 2} .
$$


Furthermore, at $T=T_{0}$ one of the following inequalities must hold:

$$
\begin{aligned}
& 1 / 2 \leq c T_{0}^{3 / 4}\left\|u_{0}\right\|_{s, 2}, \\
& 1 / 2 \leq c T_{0}^{1 / 2}\left(1+T_{0}\right)^{1 / 2^{+}}\left\|u_{0}\right\|_{s, 2},
\end{aligned}
$$

or

$$
1 / 2 \leq T_{0}\left\|u_{0}\right\|_{3 / 4,2} .
$$

Therefore, there exists a constant $M_{1}>0$ depending only on $\left\|u_{0}\right\|_{s, 2}$ such that $T=T_{0}>M_{1}$.

Moreover, for $\left\|u_{0}\right\|_{s, 2}$ sufficiently small $T=T_{0} \leq c_{s}\left\|u_{0}\right\|_{s, 2}^{-1^{-}}$.

Combining (4.19), (4.16) with (2.2), (2.12) one easily obtains (4.2), (4.4). Finally, the above remark (i.e., the right-hand side of (4.17) depends linearly on the highest derivatives) completes the proof of the lemma.

Lemma 4.2. For any $u_{0} \in H^{s}(\mathbb{R})$ with $s>3 / 4$ there exist constants $T^{*}, K>0$ such that for any $\varepsilon \in(0,1)$ the IVP (3.2) with $\alpha=2$ has a unique solution $u^{\varepsilon}(\cdot)$ in the class $C\left(\left[0, T^{*}\right]: H^{\infty}(\mathbb{R})\right)$ satisfying the estimates (4.1)-(4.5) of the previous lemma with $T^{*}$ instead of $T$.

Moreover,

$$
\left(\int_{0}^{T^{*}}\left\|D^{s+\theta \beta / 2} u^{\varepsilon}(t)\right\|_{p}^{q} d t\right)^{1 / q} \leq K
$$

for any $(\theta, \beta) \in[0,1] \times[0,1 / 2]$ with $(q, p)=(6 / \theta(\beta+1), 2 /(1-\theta))$.

Proof. Theorem 3.1 guarantees that for any $\varepsilon>0$ the IVP (3.2) has a unique solution in the class $C\left(\left[0, T_{\varepsilon}\right]: H^{\infty}(\mathbb{R})\right)$.

If $T_{\varepsilon}<T^{*} \equiv T$ (with $T$ given by Lemma 4.1) combining (4.7), (4.19) with Lemma 3.3 one finds that

$$
\sup _{\left[0, T_{\varepsilon}\right]}\left\|u^{\varepsilon}(t)\right\|_{s^{\prime}, 2} \leq c_{s^{\prime}}\left\|u_{0}^{\varepsilon}\right\|_{s^{\prime}, 2}
$$

for any $s^{\prime} \geq 0$.

Since the right-hand side of (4.21) does not depend on $T_{\varepsilon}$ (as far as $T_{\varepsilon}<$ $T^{*}$ ), we can apply Theorem 3.1 to extend the solution in the same class to the whole interval $\left[0, T^{*}\right]$.

The proof of (4.20) follows by using (2.5) in the integral equation (4.11) and then (4.16) and (4.19).

Proof of Theorem 1.1. From Lemmas 4.1 and 4.2 it follows that for any $\varepsilon \in$ $(0,1)$ the corresponding solution $u^{\varepsilon}(\cdot)$ of the IVP (3.2) with $\alpha=2$ satisfies

$$
u^{\varepsilon} \in C\left([0, T]: H^{\infty}(\mathbb{R})\right) \text {, }
$$

$$
\sup _{[0, T]}\left\|u^{\varepsilon}(t)\right\|_{s, 2} \leq K,
$$


and

$$
\int_{0}^{T}\left\|\partial_{x} u^{\varepsilon}(t)\right\|_{\infty} d t \leq K
$$

with $T$ and $K$ depending only $\left\|u_{0}\right\|_{s, 2}$.

Defining $\omega(t)=\omega^{\varepsilon, \varepsilon^{\prime}}(t)=\left(u^{\varepsilon^{\prime}}-u^{\varepsilon}\right)(t)$ for $\varepsilon>\varepsilon^{\prime}>0$, we have that $\omega(t)$ satisfies the equation

$$
\partial_{t} \omega+\partial_{x}^{3} \omega+u^{\varepsilon^{\prime}} \partial_{x} \omega+\omega \partial_{x} u^{\varepsilon}=0 .
$$

Thus, a standard argument shows that

$$
\frac{d}{d t}\|\omega(t)\|_{2} \leq c\left(\left\|\partial_{x} u^{\varepsilon}\right\|_{\infty}+\left\|\partial_{x} u^{\varepsilon^{\prime}}\right\|_{\infty}\right)(t)\|\omega(t)\|_{2}
$$

Hence,

$$
\sup _{[0, T]}\left\|\left(u^{\varepsilon^{\prime}}-u^{\varepsilon}\right)(t)\right\|_{2} \leq c\left\|u_{0}^{\varepsilon^{\prime}}-u_{0}^{\varepsilon}\right\|_{2}=o\left(\varepsilon^{s}\right)
$$

as $\varepsilon$ tends to zero.

The above inequality proves the existence and uniqueness of a strong solution $u(\cdot)$ of the IVP (1.1). To obtain the persistence property, we use the argument given by Bona and Smith in [3] to show that the $u^{\varepsilon}(\cdot)$ 's converge in $L^{\infty}\left([0, T]: H^{s}\right)$ as $\varepsilon$ tends to zero.

We shall need the following estimates:

$$
\sup _{[0, T]}\left\|u^{\varepsilon}(t)\right\|_{s+l, 2} \leq c \varepsilon^{-l},
$$

and

$$
\left(\int_{0}^{T}\left\|D^{s+\theta \beta / 2+l} u^{\varepsilon}(t)\right\|_{p}^{q} d t\right)^{1 / q} \leq c \varepsilon^{-l}
$$

for any $l>0$, with $(\theta, \beta) \in[0,1] \times[0,1 / 2],(q, p)=(6 / \theta(\alpha+1), 2 /(1+\theta))$, and where the constant $c$ depends only on $\left\|u_{0}\right\|_{s, 2}$ and $l$.

The estimate (4.26) follows from (3.3) and (4.23). The proof of (4.27) can be obtained by inserting (4.26) in the proof of Lemma 4.2 (where all the estimates depend linearly on the highest derivatives).

Now using the commutator estimates (2.13) and (2.14) in the equation (4.24) we find that

$$
\begin{aligned}
\frac{d}{d t}\|\omega(t)\|_{s, 2} \leq & c\left(\left\|\partial_{x} u^{\varepsilon^{\prime}}\right\|_{\infty}+\left\|\partial_{x} u^{\varepsilon}\right\|_{\infty}\right)(t)\|\omega(t)\|_{s, 2} \\
& +\left\|u^{\varepsilon^{\prime}}(t)\right\|_{s, 2}\left\|\partial_{x} \omega(t)\right\|_{\infty}+\left\|\partial_{x} u^{\varepsilon}(t)\right\|_{s, r_{1}}\|\omega(t)\|_{r_{2}}
\end{aligned}
$$

with $\frac{1}{r_{1}}+\frac{1}{r_{2}}=\frac{1}{2}$. Since $s>3 / 4$, combining the estimates (4.22), (4.23), and (4.25)-(4.27) with the Sobolev embedding theorem and the three line theorem (see [1]) it follows that:

$$
\int_{0}^{T}\left\|u^{\varepsilon^{\prime}}(t)\right\|_{s, 2}\left\|\partial_{x} \omega(t)\right\|_{\infty} d t \leq c \int_{0}^{T}\left\|\partial_{x} \omega(t)\right\|_{\infty} d t=o(1)
$$


and

$$
\int_{0}^{T}\left\|\partial_{x} u^{\varepsilon}(t)\right\|_{s, r_{1}}\|\omega(t)\|_{r_{2}} d t=o(1)
$$

as $\varepsilon$ tends to zero, for $r_{1}$ sufficiently large.

Thus after applying Gronwall's inequality in (4.28) we obtain that

$$
\sup _{[0, T]}\left\|\left(u^{\varepsilon^{\prime}}-u^{\varepsilon}\right)(t)\right\|_{s, 2}=o(1) \quad \text { as } \varepsilon \rightarrow 0 .
$$

This proves that the IVP $(1.1)$ has a unique solution $u \in C\left([0, T]: H^{s}(\mathbb{R})\right)$ whenever $u_{0} \in H^{s}(\mathbb{R})$ with $s>3 / 4$ (i.e., existence, persistence, and uniqueness).

As it is well known (see $[3,19,27]$ ), the approach of using $u^{\varepsilon}(\cdot)$-solutions has the advantage that it provides continuous dependence almost automatically. Therefore, to complete the proof of Theorem 1.1, we shall restrict ourselves to showing that the $u^{\varepsilon}(\cdot)$-solutions converge to $u$ in the norms described in (1.13)-(1.15).

For this purpose we use that the $\omega(\cdot)$ solution of $(4.24)$ satisfies the following integral equation:

$$
\omega(t)=\left(u^{\varepsilon^{\prime}}-u^{\varepsilon^{\prime}}\right)(t)=U^{2}(t) \omega_{0}-\int_{0}^{T} U^{2}(t-\tau)\left(u^{\varepsilon^{\prime}} \partial_{x} \omega+\omega \partial_{x} u^{\varepsilon}\right)(\tau) d \tau .
$$

Using the notation

$$
\begin{aligned}
& \lambda(v, T, l)=\left(\int_{0}^{T}\left\|J^{l} \partial_{x} v(t)\right\|_{\infty}^{4} d t\right)^{1 / 4}, \\
& \sigma(v, T, l)=\sup _{x}\left(\int_{0}^{T}\left|D^{l} \partial_{x} v(x, t)\right|^{2} d t\right)^{1 / 2}, \\
& \nu(v, T, l)=\left(\int \sup _{[0, T]}\left|J^{l} u(x, t)\right|^{2} d x\right)^{1 / 2},
\end{aligned}
$$

the estimates (4.20), (4.22), (4.23), (4.29), and (4.30), and arguing as in the proof of Lemma 4.1 it is not hard to see that

$$
\begin{aligned}
\max & \left\{\lambda(\omega, T, r) ; \sigma(\omega, T, s) ;(1+T)^{1 / 4^{+}} \nu\left(\omega, T, r^{\prime}\right)\right\} \\
& \leq o(1)+c T^{1 / 2}\left(\nu\left(u^{\varepsilon}, T, 0\right) \sigma(\omega, T, s)+\nu(\omega, T, 0) \sigma\left(u^{\varepsilon}, T, s\right)\right)
\end{aligned}
$$

for any $\left(r, r^{\prime}\right) \in[0, s-3 / 4] \times[0, s-3 / 4)$.

From (4.18) and (4.19) it follows that

$$
\max \left\{\lambda(\omega, T, r) ; \sigma(\omega, T, s) ;(1+T)^{-1 / 4} \nu\left(\omega, T, r^{\prime}\right)\right\}=o(1)
$$

as $\varepsilon$ tends to zero, which combined with (2.5) finishes the proof.

Remark. Notice that the above proof also establishes the uniqueness and regularity of strong solutions (i.e., solutions that are limits of classical ones in $\left.C\left([0, T]: H^{\infty}\right)\right)$. 


\section{Proof of Theorem 1.3}

The proof of this theorem has some differences from the proof provided in the previous section for Theorem 1.1. In this case $\alpha \in[1,2)$, thus the gain of derivatives $\alpha / 2$ in the local smoothing effect (2.2) is smaller than one. Hence, the integral equation cannot be used (as in the previous case $\alpha=2$ ) to establish this effect. To obtain it one has to rely on the differential equation, which yields a slightly weaker version of this result. Consequently, we have to use the stronger estimate (1.8) for the associated maximal function instead of the simpler one in (1.7) to balance this loss of information (see (5.10)).

As in the previous case the main idea in the proof is to obtain an a priori estimate of the $L^{\infty}\left([0, T]: H^{s}\right)$-norm of the solutions $u^{\varepsilon}(\cdot)$ of the IVP (3.2) with the bound $M$ and the time $T$ independent of $\varepsilon$.

We begin by proving the following version of the local smoothing effect.

Lemma 5.1. Let $u=u^{\varepsilon} \in C\left([0, T]: H^{\infty}\right)$ be a solution of the IVP (3.2) with $\alpha \in[1,2)$. Then

$$
\begin{aligned}
& \left(\int_{0}^{T} \int\left|D^{s_{0}} \partial_{x} u(x, t)\right|^{2} \chi_{j}^{\prime}(x) d x d t\right)^{1 / 2} \\
& \leq c\left\|u_{0}\right\|_{s, 2} \cdot\left(1+T+\int_{0}^{T}\left\|\partial_{x} u(t)\right\|_{\infty} d t+T\left\|u_{0}\right\|_{l, 2}\right) \\
& \quad \times \exp \left(c \int_{0}^{T}\left\|\partial_{x} u(t)\right\|_{\infty} d t\right)
\end{aligned}
$$

where $s_{0}=s+\alpha / 2-1, l>1 / 2, \chi$ denotes a nondecreasing smooth function such that $\chi^{\prime}$ is supported in $(-1,2)$ with $\chi^{\prime}=1$ on $[0,1]$ and $\chi_{j}(\cdot)=$ $\chi(\cdot-j)$, and the constant $c$ is independent of $\varepsilon$.

To establish (5.1) we need, in addition to Lemma 2.10, some commutator estimates. First we have the Calderón commutator theorem.

Theorem 5.2. Let $g: \mathbb{R} \rightarrow \mathbb{R}$ be a $C^{\infty}$ function with $g^{\prime} \in L^{\infty}$. Then the operator $[\sigma ; g] \partial_{x}$ maps $L^{2}(\mathbb{R})$ into $L^{2}(\mathbb{R})$ with

$$
\left\|[\sigma ; g] \partial_{x} f\right\|_{2} \leq c\left\|g^{\prime}\right\|_{\infty}\|f\|_{2} .
$$

Proof. [4], see also [6, Theorem 35].

Next we state an estimate, due to Ginibre and Velo [11], concerning the local smoothing effect (1.5) in solutions of the IVP (1.2) with $u_{0} \in L^{2}$ and $u_{0} \in H^{\alpha}$.

Lemma 5.3. Let $h \in C^{\infty}(\mathbb{R})$ with $h^{\prime}$ having compact support. Then for $\alpha \in$ $[1,2)$

$$
2 \int f\left(D^{\alpha} \partial_{x} f\right) h=(1+\alpha) \int\left(D^{\alpha / 2} f\right)^{2} h^{\prime}+2 \int f R_{\alpha}(h) f,
$$

where $\left\|R_{\alpha}(h) f\right\|_{2} \leq c_{\alpha}\left\|\widehat{D^{\alpha} h^{\prime}}\right\|_{1}\|f\|_{2}$.

Proof. See [11, Proposition 2.1 and Remark (14)]. 
Proof of Lemma 5.1. From the equation in (3.2) one has that

$$
\partial_{t} A u-D^{\alpha} \partial_{x} A u+u \partial_{x} A u+[A ; u] \partial_{x} u=0
$$

where $A \equiv D^{S_{0}-\alpha / 2} \partial_{x}$. Multiplying (5.4) by $-A u \chi_{j}(x)$, integrating in the space variable, and using (5.3) after integration by parts we find that

$$
\begin{aligned}
& -\frac{d}{d t} \int(A u)^{2} \chi_{j}+(1+\alpha) \int\left(D^{s_{0}} \partial_{x} u\right)^{2} \chi_{j}+\int A u \cdot R_{\alpha}\left(\chi_{j}\right) A u \\
& \quad+2 \int\left(\partial_{x} u \chi_{j}+u \chi_{j}^{\prime}\right)(A u)^{2} \\
& \quad-2 \int[A ; u] \partial_{x} u A u \chi_{j}=0 .
\end{aligned}
$$

The estimates (2.13), (2.14), and (5.2) lead to the following inequalities

$$
\begin{aligned}
\left\|[A ; u] \partial_{x} u\right\|_{2} & \leq\left\|\sigma\left[D^{s} ; u\right] \partial_{x} u\right\|_{2}+\left\|[\sigma, u] \partial_{x} D^{s} u\right\|_{2} \\
& \leq c\left(\|u\|_{\infty}+\left\|\partial_{x} u\right\|_{\infty}\right)\|u\|_{s, 2} .
\end{aligned}
$$

Hence, integrating the identity $(5.5)$ in the time interval $[0, T]$ we obtain

$$
\begin{aligned}
& \int_{0}^{T} \int\left|D^{s_{0}} \partial_{x} u(x, t)\right|^{2} \chi_{j}^{\prime}(x) d x d t \\
& \quad \leq c \sup _{[0, T]}\|u(t)\|_{s, 2}^{2}\left(1+T+\int_{0}^{T}\left\|\partial_{x} u(t)\right\|_{\infty} d t+T \sup _{[0, T]}\|u(t)\|_{l, 2}\right)
\end{aligned}
$$

for any $l>1 / 2$, which combined with the energy estimate (3.3) yields the result.

Proof of Theorem 1.3. To simplify the exposition we introduce the following notation:

$$
\begin{gathered}
\mu(T)=\left(\sum_{j=-\infty}^{\infty} \sup _{[0, T]} \sup _{j \leq x<j+1}|u(x, t)|^{2}\right)^{1 / 2}, \\
\lambda(T)=\left(\int_{0}^{T}\left\|\partial_{x} u(t)\right\|_{\infty}^{4} d t\right)^{1 / 4}, \\
\gamma(T)=\sup _{[0, T]}\|u(t)\|_{s, 2} .
\end{gathered}
$$

Thus, using Hölder's inequality, the energy estimate (3.3) can be written as

$$
\gamma(T) \leq c_{s}\left\|u_{0}\right\|_{s, 2} \exp \left(c T^{3 / 4} \lambda(T)\right) .
$$

Now from the integral equation

$$
u(t)=U^{\alpha}(t) u_{0}-\int_{0}^{T} U^{\alpha}(t-\tau) u \partial_{x} u(\tau) d \tau
$$


and the linear estimates $(2.5)$ (with $(\theta, \beta)=(1,(\alpha-1) / 2))$, and (2.11) one easily sees that

$$
\lambda(T) \leq c\left\|D^{(5-\alpha) / 4} u_{0}\right\|_{2}+c \int_{0}^{T}\left\|D^{(5-\alpha) / 4}\left(u \partial_{x} u\right)(t)\right\|_{2} d t
$$

and

$$
\mu(T) \leq c(1+T)^{\rho}\left\{\left\|u_{0}\right\|_{s_{0}, 2}+c \int_{0}^{T}\left\|\left(u \partial_{x} u\right)(t)\right\|_{s_{0}, 2} d t\right\}
$$

for any $\rho>3 / 4$ and $s_{0}=s+\alpha / 2-1>(\alpha+1) / 4$.

Since $\alpha<2$, and $s \geq(9-3 \alpha) / 4$, we can assume that $s_{0} \geq(5-\alpha) / 4$. To bound the integral terms in the right-hand sides of (5.8) and (5.9) we use (2.13), (2.14), and (5.7) to obtain the following string of inequalities:

$$
\begin{aligned}
& \int_{0}^{T}\left\|\left(u \partial_{x} u\right)(t)\right\|_{s_{0}, 2} d t \leq c T^{1 / 2}\left\{\int_{0}^{T} \int\left|J^{s_{0}}\left(u \partial_{x} u\right)(x, t)\right|^{2} d x d t\right\}^{1 / 2} \\
& \leq c T^{1 / 2}\left\{\left(\int_{0}^{T} \int\left|u D^{s_{0}} \partial_{x} u\right|^{2} d x d t\right)^{1 / 2}\right. \\
& \left.+\left(\int_{0}^{T}\left(\|u\|_{\infty}^{2}+\left\|\partial_{x} u\right\|_{\infty}^{2}\right)\|u\|_{s_{0}, 2}^{2}(t) d t\right)^{1 / 2}\right\} \\
& \leq c T^{1 / 2}\left(\sum_{j} \int_{0}^{T} \int\left|u D^{s_{0}} \partial_{x} u\right|^{2} \chi_{j}^{\prime} d x d t\right)^{1 / 2}+c T^{3 / 4} \sup _{[0, T]}\|u(t)\|_{s_{0}, 2} \\
& +\left(\int_{0}^{T}\left\|\partial_{x} u(t)\right\|_{\infty}^{4} d t\right)^{1 / 4}+c T \sup _{[0, T]}\|u(t)\|_{s_{0}, 2} \sup _{[0, T]}\|u(t)\|_{l, 2} \\
& \leq c T^{1 / 2}\left\{\sum_{j} \sup _{[0, T]} \sup _{j \leq x<j+1}|u(x, t)|^{2}\right\}^{1 / 2} \\
& \times\left\{\sup _{j} \int_{0}^{T} \int\left|D^{s_{0}} \partial_{x} u\right|^{2} \chi_{j}^{\prime} d x d t\right\}^{1 / 2} \\
& +c T^{3 / 4}\left\|u_{0}\right\|_{s_{0}, 2} \lambda(T) \exp \left(c T^{3 / 4} \lambda(T)\right) \\
& +c T\left\|u_{0}\right\|_{s_{0}, 2}\left\|u_{0}\right\|_{l, 2} \exp \left(c T^{3 / 4} \lambda(T)\right) \\
& \leq c T^{1 / 2} \mu(T)\left\|u_{0}\right\|_{s, 2} \exp \left(c T^{3 / 4} \lambda(T)\right)\left(1+T+T^{3 / 4} \lambda(T)+T\left\|u_{0}\right\|_{l, 2}\right) \\
& +c T^{3 / 4}\left\|u_{0}\right\|_{s_{0}, 2} \exp \left(c T^{3 / 4} \lambda(T)\right)\left(\lambda(T)+T^{1 / 4}\left\|u_{0}\right\|_{l, 2}\right) \\
& =c\left\|u_{0}\right\|_{s, 2} \exp \left(c T^{3 / 4} \lambda(T)\right)\left(T^{1 / 2} \mu(T)+1\right) \\
& \times\left(1+T+T^{3 / 4} \lambda(T)+T\left\|u_{0}\right\|_{l, 2}\right) \\
& \equiv E(T) \text {, }
\end{aligned}
$$

where $s_{0}=s+\alpha / 2-1<s$ and $l>1 / 2$. 
Therefore, combining (5.8)-(5.10) we have that

$$
\max \left\{\lambda(T) ;(1+T)^{-\rho} \mu(T)\right\} \leq c\left\|u_{0}\right\|_{s, 2}+E(T) .
$$

Now we define $T_{0}$ by the expression

$$
\max \left\{c T_{0}^{3 / 4} \lambda\left(T_{0}\right) ; c T_{0}^{1 / 2} \mu\left(T_{0}\right)\right\}=1 / 2 .
$$

Hence, for $T \leq T_{0}$

$$
\max \left\{\lambda(T) ;(1+T)^{-\rho} \mu(T)\right\} \leq c\left\|u_{0}\right\|_{s, 2}\left(1+T+T\left\|u_{0}\right\|_{l, 2}\right) .
$$

Moreover, at least one of the following inequalities holds:

$$
\frac{1}{2} \leq c T_{0}^{3 / 4}\left\|u_{0}\right\|_{s, 2}\left(1+T+T\left\|u_{0}\right\|_{l, 2}\right)
$$

or

$$
\frac{1}{2} \leq c(1+T)^{\rho}\left\|u_{0}\right\|_{s, 2}\left(1+T+T\left\|u_{0}\right\|_{l, 2}\right) .
$$

Thus, we conclude that there exists $T=T_{0} \geq M=M\left(\left\|u_{0}\right\|_{s, 2}\right)>0$ such that $\lambda(T), \mu(T)$, and $\gamma(T)$, and consequently the term in the left-hand side of (5.1), can be bounded by a constant $K$ with $K$ and $T$ dependent but only on $\left\|u_{0}\right\|_{s, 2}$.

At this point the rest of the proof follows the argument used in the previous section (see also $[19,27])$. Therefore it will be omitted.

\section{REFERENCES}

1. T. B. Benjamin, Internal waves of permanent form in fluid of great depth, J. Fluid Mech. 29 (1967), 559-592.

2. J. L. Bona and R. Scott, Solutions of the Korteweg-de Vries equation in fractional order Sobolev spaces, Duke Math. J. 43 (1976), 87-99.

3. J. L. Bona and R. Smith, The initial value problem for the Korteweg-de Vries equation, Proc. Roy. Soc. London Ser. A 278 (1978), 555-601.

4. A. P. Calderón, Commutators of singular integral operators, Proc. Nat. Acad. Sci. U.S.A. 53 (1965), 1092-1099.

5. L. Carleson, Some analytical problems related to statistical mechanics, Euclidean Harmonic Analysis, Lecture Notes in Math, vol. 779, Springer-Verlag, Berlin and New York, 1979, pp. 9-45.

6. R. R. Coifman and Y. Meyer, Au delá des opérateurs pseudodifféntieles, Astérisque $\mathbf{5 7}$ (1978).

7. __ Nonlinear harmonic analysis, operator theory and P.D.E., Beijing Lectures in Harmonic Analysis, Princeton Univ. Press, Princeton, NJ, 1986, pp. 3-45.

8. P. Constantin and J. C. Saut, Local smoothing properties of dispersive equations, J. Amer. Math. Soc. 1 (1988), 413-446.

9. B. Dahlberg and C. E. Kenig, A note on the almost everywhere behavior of solutions to the Schrödinger equation, Harmonic Analysis, Lecture Notes in Math., vol. 908, Springer-Verlag, Berlin and New York, 1982, pp. 205-208. 
10. J. Ginibre and G. Velo, Scattering theory in the energy space for a class of nonlinear Schrödinger equation, J. Math. Pures Appl. 64 (1985), 363-401.

11. _ Commutator expansions and smoothing properties of generalized BenjaminOno equations, Ann. Inst. H. Poincaré Phys. Théor. 51 (1989), 221-229.

12. _ Smoothing properties and existence of solutions for the generalized BenjaminOno equation, preprint .

13. J. Ginibre and Y. Tsutsumi, Uniqueness for the generalized Korteweg-de Vries equations, SIAM J. Math. Anal. 20 (1989), 1388-1425.

14. T. Kato, Quasilinear equations of evolutions, with applications to partial differential equations, Lectures Notes in Math., vol. 448, Springer-Verlag, Berlin and New York, 1975, pp. 27-50.

15. _ On the Korteweg-de Vries equation, Manuscripta Math. 29 (1979), 89-99.

16. __ On the Cauchy problem for the (generalized) Korteweg-de Vries equation, Adv. Math. Suppl. Stud., Stud. Appl. Math. 8 (1983), 93-128.

17. T. Kato and G. Ponce, Commutator estimates and the Euler and Navier-Stokes equations, Comm. Pure Appl. Math. 41 (1988), 891-907.

18. C. E. Kenig, G. Ponce and L. Vega, On the (generalized) Korteweg-de Vries equation, Duke Math. J. 59 (1989), 585-610.

19. _ Oscillatory integrals and regularity of dispersive equations, Indiana Univ. Math. J. (to appear).

20. C. E. Kenig and A. Ruiz, A strong type $(2,2)$ estimate for the maximal function associated to the Schrödinger equation, Trans. Amer. Math. Soc. 280 (1983), 239-246.

21. D. J. Korteweg and G. de Vries, On the change of form of long waves advancing in a rectangular canal, and on a new type of long stationary waves, Philos. Mag. 39 (1895), 422-443.

22. S. N. Kruzhkov and A. V. Faminskii, Generalized solutions of the Cauchy problem for the Korteweg-de Vries equation, Math. USSR-Sb. 48 (1984), 93-138.

23. B. Marshall, Mixed norm estimates for the Klein-Gordon equation, Proc. Conf. in Harmonic Analysis in honor of A. Zygmund, Wadsworth Intl. Math. Series, 1981, pp. 638-639.

24. R. M. Miura, The Korteweg-de Vries equation: a survey of results, SIAM Rev. 18 (1976), 412-459.

25. H. Ono, Algebraic solitary waves in stratified fluids, J. Phys. Soc. Japan 39 (1975), 10821091.

26. H. Pecher, Nonlinear small data scattering for the wave and Klein-Gordon equation, Math. Z. 185 (1985), 261-270.

27. G. Ponce, On the global wellposedness of the Benjamin-Ono equation, Differential Integral Equations (to appear).

28. G. Ponce and L. Vega, Nonlinear small data scattering for the generalized Korteweg-de Vries equation, J. Funct. Anal. 90 (1990), 445-457.

29. J. C. Saut, Sur quelque généralisations de l'equation de Korteweg-de Vries, J. Math. Pure Appl. 58 (1979), 21-61.

30. J. C. Saut and R. Temam, Remarks on the Korteweg-de Vries equation, Israel J. Math. 24 (1976), 78-87.

31. P. Sjölin, Regularity of solutions to the Schrödinger equation, Duke Math. J. 55 (1987), 699-715.

32. E. M. Stein, Oscillatory integrals in Fourier analysis, Beijing Lectures in Harmonic Analysis, Princeton Univ. Press, Princeton, NJ, 1986, pp. 307-355.

33. E. M. Stein and G. Weiss, Introduction to Fourier analysis in eucliden spaces, Princeton Univ. Press, Princeton, NJ.

34. R. S. Strichartz, Restriction of Fourier transform to quadratic surfaces and decay of solutions of wave equations, Duke Math. J. 44 (1977), 705-714. 
35. P. Tomas, A restriction theorem for the Fourier transform, Bull. Amer. Math. Soc. 81 (1975), 477-478.

36. L. Vega, Schrödinger equations: pointwise convergence to the initial data, Proc. Amer. Math. Soc. 102 (1988), 874-878.

37. __ Doctoral thesis, Universidad Autonoma, Madrid, Spain, 1987.

(C. Kenig and L. Vega) Department of Mathematics, University of Chicago, Chicago, ILLINOIS 60637

Current address, L. Vega: Facultad de Ciencias, Universidad Autonoma de Madrid, Cantobianco, Madrid (28049), Spain

(G. Ponce) Department of Mathematics, Pennsylvania State University, University Park, Pennsylvania 16802 